\title{
Applying a Meta-Analysis to Daily Clinical Practice
}

\author{
William F. Miser $M D, M A$
}

You are seeing a cranky 4-year-old boy who bas a low-grade fever and symptoms of an upper respiratory tract infection. On examination be appears nontoxic but definitely bas a left otitis media. He bas previously been bealtby and bas no bistory of an ear infection. You want to prescribe antibiotics, but family finances are tight (both parents are laid off from work), and the child bates to take any type of medicine. As you discuss the situation with the parents, you remember an article published recently that suggested childhood otitis media could be effectively treated with a shorter course of antibiotics. You write a prescription for a 5-day course of amoxicillin and tell the parents you will telephone toward the end of treatment to determine whether a longer course is needed. In the meantime, you find the article titled "Treatment of Acute Otitis Media With a Shortened Course of Antibiotics - A Meta-Analysis." You bave seen the term meta-analysis before but are unsure bow to critique the article.

The ability to evaluate an article critically is a necessary skill for clinicians to maintain competency and to provide patients with the best possible care. ${ }^{2}$ Although the first reported attempt at a meta-analysis was in $1904,{ }^{3}$ it was not until the mid-1970s when this actual methodologic technique was defined. ${ }^{4,5}$ Since then, the biostatistical method known as meta-analysis has gained in popularity. The number of published articles using this technique has increased from 109 in 1988 to 754 in 1998. Despite its widespread use, meta-analysis continues to be a controversial method. ${ }^{6}$

The intent of this article is to help you understand the purpose, strengths, and weaknesses of a meta-analysis, and to provide you with the tools needed to critically evaluate an article that uses this methodology. Because of space limitations, I can not cover everything in exhaustive detail, and the reader is encouraged to refer to the references for several outstanding reviews. Using the article mentioned in the case as an example, you will learn how to use a worksheet that allows you to assess the validity of a meta-analysis. The ultimate goal is to enable you to determine whether you should apply

Submitted, revised, 8 March 1999.

From the Department of Family Medicine, The Ohio State University, Columbus. Address reprint requests to William F. Miser, MD, MA, Rardin Family Practice Center, 2231 North High St, Columbus, OH 43201.

This article was reprinted with permission from Miser WF. Applying a meta-analysis to daily clinical practice. In Geyman JP, Deyo RA, Ramsey SD, editors. Evidence-based clinical practice: concepts and approaches. Boston: Butterworth-Heinemann, 2000:57-64. the findings of a meta-analysis to the daily clinical problems you encounter.

\section{Characteristics of a Meta-Analysis}

The medical literature consists of two major types of studies: (1) those that report original research (analytic, primary studies), and (2) those that summarize or draw conclusions from this original research (integrative, secondary studies). There are various types of integrative studies. The simplest and least stringent study is a nonsystematic review written as a continuing medical education article by authors expert in a subject matter. These articles may be based solely on opinions and clinical experiences, with a cursory, if any, literature review being performed. References used for support are mainly review articles themselves. The authors might pick and choose the studies that support their argument, while ignoring those that disagree. Oxman and Guyatt ${ }^{7}$ found that the greater the expertise of the reviewer, the lower the quality of the review. For example, a review article on screening for prostate cancer written by a urologist might reach a completely different conclusion than one written by a family physician, although the literature available to both is the same. This type of subjective review article is fraught with bias and can be misleading. ${ }^{8}$

A systematic review is a more precise integrative study. This type of review article provides an overview of original research using a precise protocol with a statement of objectives and a literature review conducted according to a specific and repro- 
A research question is precisely defined.

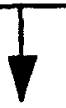

A protocol is established before the study begins which states the objectives of the review of RCT's and outlines the eligibility criteria for these RCT's.

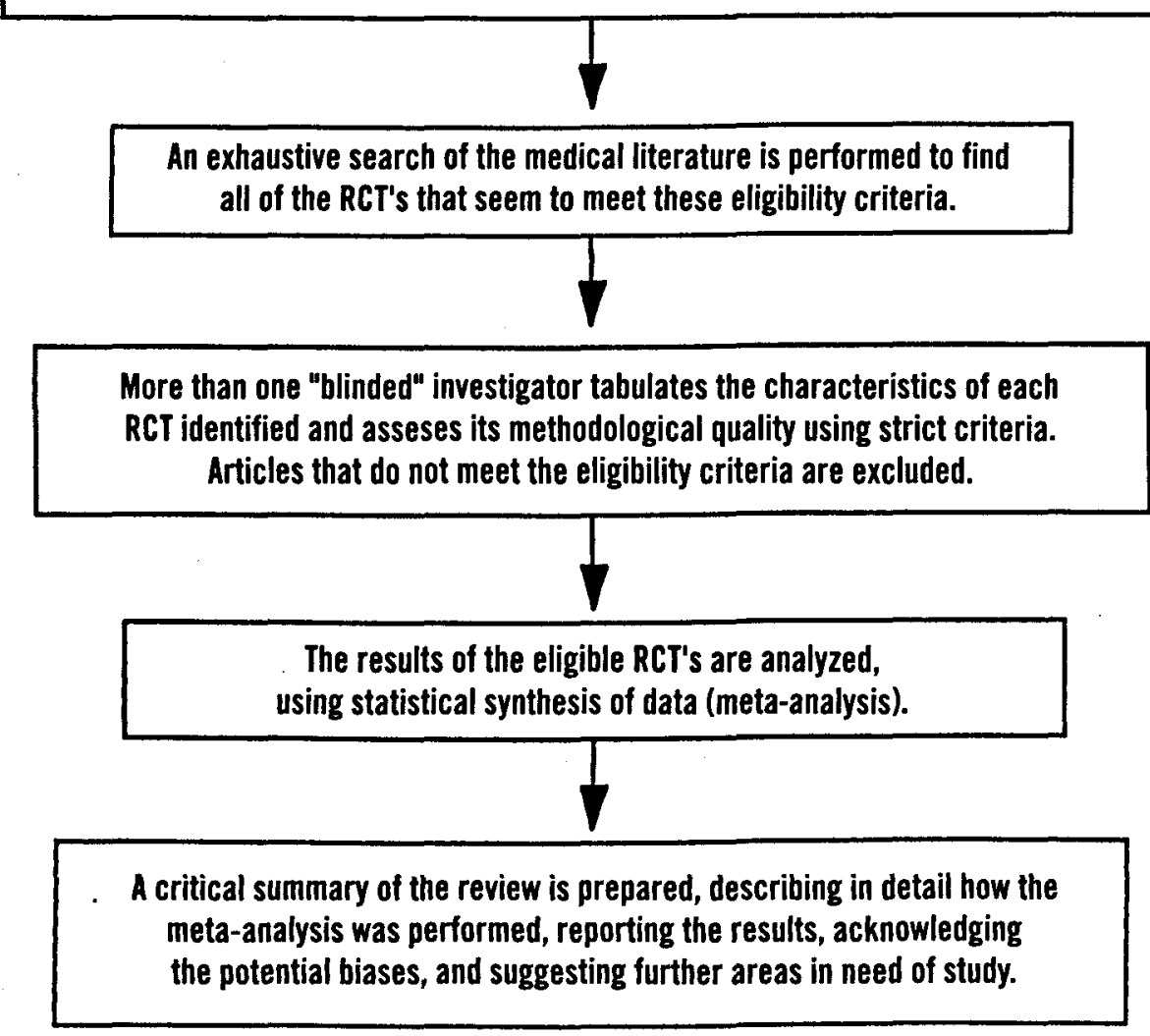

Figure 1. Steps in conducting a meta-analysis. RCTs = randomized clinical trials.

ducible methodology. ${ }^{9-13}$ The author provides a detailed description of how the articles were obtained and the methods by which articles were included and excluded for consideration. Much like conducting original research, the author follows a protocol in researching primary studies. When assessing such a review, the reader can judge the quality of science that went into the writing of the review.

A meta-analysis is a type of systematic review of the literature that includes combining and analyzing the data of individual trials. As would any other research project, a meta-analysis should have a detailed written protocol prepared in advance that includes a narrowly focused question to be answered. Original studies are found that address this question, their data are combined, statistical tests are applied to these combined results, and the re- sults are reported (Figure 1). The meta-analysis is a statistical procedure that integrates the results of several independent clinical trials considered by the analyst to be "combinable." 14 So, in addition to critically reviewing the primary research, the author also statistically combines the results. It literally is a study of studies. ${ }^{15} \mathrm{~A}$ meta-analysis has been likened to an observational study of the evidence. ${ }^{14}$ Most meta-analyses are applied to randomized clinical trials (RCTs), although recently some have attempted, with varying degrees of success, to apply this technique to nonrandomized trials and epidemiologic research. ${ }^{16-18}$

The potential benefits of meta-analyses are found in Table 1. The sample in a RCT might be too small to detect a difference between groups when, in reality, there is a difference (type II error). In a review of RCTs with negative results published 
Table 1. Potential Benefits of a Meta-Analysis.

1. Increase the number of subjects in an analysis, thus improving the statistical power to detect overall differences for primary end-points within subgroups

2. Provide more objective appraisal of the literature, which might resolve uncertainty when reports disagree

3. Improve estimates of effect size or magnitude of association

4. Address research questions not posed at the start of individual trials

5. Assimilate large amounts of information

6. Improve the quality of primary research

Adapted from Chalmers et al, ${ }^{5}$ Peipert and Bracken, ${ }^{13}$ and Sacks et al. ${ }^{16}$

between 1975 to 1990 in $7 A M A$, Lancet, and New England Fournal of Medicine, the vast majority of these trials had too few patients, which led to insufficient power to detect a $25 \%$ or $50 \%$ difference. ${ }^{19} \mathrm{~A}$ major advantage of a meta-analysis is that it can combine several smaller studies, thus increasing the number of subjects in the final analysis and subsequently improving the power to detect a treatment effect if one is present.

Another potential benefit of a meta-analysis is that it might answer questions about whether an overall study result differs among various subgroups (eg, among older or younger patients, men and women, or subjects with varying degrees of disease severity). ${ }^{6}$ Also, if done well, a meta-analysis is an excellent critical review of the literature that points out the strengths and weaknesses of previous research on a given topic. As such, it can generate research questions to be addressed in future studies, along with a more accurate sample size needed to answer these questions.

A meta-analysis is only as good as the research on which it is based, however. It is not an infallible tool. ${ }^{6}$ Unfortunately, many authors view the metaanalysis as an easy way to get published, especially with the help of software programs that allow rapid statistical calculations. ${ }^{20}$ As a result, there is growing concern about the quality of most meta-analyses. ${ }^{16}$ The three main threats to the validity of a meta-analysis are (1) combining studies that are dissimilar, (2) publication bias, and (3) including poor-quality studies in the analysis. Because of these threats, many meta-analyses are methodologically flawed. ${ }^{16,21,22}$ A classic example of a flawed meta-analysis is one which suggested that giving intravenous magnesium was beneficial to those who had myocardial infarctions. ${ }^{23}$ A large trial involving
58,000 patients (International Study of Infarct Survival-4), however, found no benefit. ${ }^{24}$ On further review, the meta-analysis was misleading because of publication bias and methodologic weaknesses in smaller trials.

So, in summary, meta-analyses have great potential to be useful for clinicians, but not all are methodologically sound. As such, it is important to have the basic skills to be able to distinguish a well-designed meta-analysis from one that should be recycled.

\section{Critical Appraisal of a Meta-Analysis}

Assessing a meta-analysis involves two steps: (1) conduct an initial validity and relevance screening test, which has been described in detail, ${ }^{2}$ and if the meta-analysis passes the screening test, (2) perform a more in-depth evaluation of its validity.

\section{Step One - Conduct an Initial Validity and Relevance Screening Test}

The first step when assessing a meta-analysis is to ask yourself, "Is this article worth taking the time to review in depth?" This step can be quickly accomplished by examining the six simple questions outlined in Table 2. ${ }^{2}$ Using the article mentioned in the case and the questions in Table 2, you find these answers. The article you are assessing is published in $7 A M A$. You are almost certain that this journal is peer-reviewed, which is confirmed in the Instructions for Authors (" $7 A M A$ is an international, peer-reviewed, general medical journal..."). You look at the end of the article to find that funding support was from two foundations, but none from a company that has commercial interests in the drugs mentioned in the study. Your attention now turns to the abstract. In the introduction, you read the purpose of the meta-analysis was to determine whether treating children with a shorter course of antibiotics is comparable to treating them with a longer course. You then read this conclusion: "[F]ive days of short-acting antibiotic use is effective treatment for uncomplicated acute otitis media in children." You scan the rest of the abstract and find that the outcomes were treatment failures, relapses, or reinfections. Taking antibiotics for a shorter length of time will reduce costs and lessen the chances of adverse reactions and drug resistance, outcomes that are important to you and your patients. As a primary care physician, otitis media is 
Is this article worth taking the time to review in depth?

1. Is the article from a peer-reviewed journal? Articles published in a peer-reviewed journal bave already gone througb an extensive review and editing process

2. Is the location of the study similar to mine so the results, if valid, would apply to my practice?

3. Is the study sponsored by an organization that might influence the study design or results?

Read the conclusion of the abstract to determine relevance

4. Will this information, if true, have a direct impact on the health of my patients, and is it something they will care about?

5. Is the problem addressed one that is common to my practice, and is the intervention or test feasible and available to me?

6. Will this information, if true, require me to change my current practice?

\begin{tabular}{cc}
$\begin{array}{c}\text { Yes } \\
\text { (go on) }\end{array}$ & $\begin{array}{c}\text { No } \\
\text { (stop) }\end{array}$ \\
Yes \\
(go on) & No \\
Yes & (stop) \\
(pause) & No \\
& (go on) \\
Yes & No \\
(go on) & (stop) \\
Yes & No \\
(go on) & (stop) \\
Yes & No \\
(go on) & (stop) \\
\hline
\end{tabular}

Adapted from Table 1 in Miser. ${ }^{2}$

Source: Questions 4 through 6 were adapted from Slawson D, Shaughnessy A, Ebell M, Barry H: mastering medical information and the role of POEMs: patient-oriented evidence that matters. J Fam Pract 1997;45:195-6.

Note: A stop or pause answer to any of the following should prompt you to seriously question whether you should spend the time to review the article critically.

one of the most common problems encountered in your practice, and you could easily write a prescription for a shorter course of antibiotics. Because you currently treat otitis media in a child with at least 10 days of antibiotics, using this information will require you to change your current practice.

In only a few seconds you have quickly answered yes to six pertinent questions that allow you to decide whether you want to take the time to review this meta-analysis critically. This tool allows you to weed out and recycle those articles that are not relevant to your practice, thus allowing more time to examine the validity of those few articles that might have an impact on the care of your patients. Based on the information you have found from this screening tool, you might be tempted to begin writing prescriptions for a shorter course of antibiotics for otitis media. Before you make a drastic change in your prescribing pattern for this common problem, however, you want assurances that the authors of the paper conducted a valid meta-analysis.

\section{Step Two - Determine the Validity of the Meta- Analysis}

You decide to assess this JAMA article critically based on your initial screening results. Using the questions found in Table 3, you now turn your attention to the Methods section.

1. Was the literature searcb done well? The strength of a meta-analysis depends on the quality of the medical literature search. This search must be thorough and objective, using multiple comput- erized literature databases and various techniques. ${ }^{25}$ A poorly done literature search often will result in a meta-analysis that yields invalid conclusions. The authors should attempt to do more than a simple MEDLINE search; even the best MEDLINE search will miss up to $20 \%$ to $70 \%$ of articles pertinent to the topic. ${ }^{16,26-28}$ In addition to a computerized literature search, the authors should review the references of each of the articles found through the search. Looking up citations of these references often yields useful articles not selected in the original search.

Publication bias is a major threat to the validity of a meta-analysis. ${ }^{29}$ Studies showing statistically significant (positive) results and having larger sample sizes are more likely to be written and submitted by authors and subsequently accepted and published than are nonsignificant (negative) studies. ${ }^{30-33}$ For example, in one study of publication bias, $85 \%$ of studies with significant results compared with $65 \%$ of negative studies had been published after 10 years. ${ }^{34}$ The median time to publication was 4.8 years for the studies with significant results compared with 8.0 years for those with negative results.

To avoid this bias, investigators should decide whether the search should include unpublished data known as the gray literature. ${ }^{11}$ Talking to experts in the research community and reviewing research data that have not yet been published could yield results different from those published. Obtaining and including unpublished data might help avoid publication bias, but not totally. ${ }^{35}$ The 
Was the literature search done well?

1. Was it comprehensive

2. Were the search methods systematic and clearly described?

Yes

Yes

Yes

Yes

4. Was the issue of publication bias addressed?
No

No

No

No

Yes No

Yes No

Yes No

Yes No

Yes No

Yes No

Yes No

4. Was the selection process reliable? (Were at least two independent selectors used? Was the extent of selection disagreement evaluated?)

Was the quality of the primary studies evaluated?

1. Did all studies, published or not, have the same standard applied?

2. Were at least 2 independent evaluators used, and was the inter-rater agreement assessed and adequate?

3. Were the evaluators blinded to the authors, institutions, and results of the primary studies?

Were results from the studies combined appropriately?

1. Were the studies similar enough to combine results (were the study designs, populations, exposures, outcomes, and direction of effect similar in the studies that are combined?)

2. Was a test for heterogeneity done and was its $P$ value nonsignificant?

Yes

No

Yes No

Yes

No

Yes

No

Yes

No

Was a statistical combination (meta-analysis) done properly?

1. Were the methods of the studies similar?

2. Was the possibility of chance differences statistically addressed (was a test for homogeneity done?

3. Are appropriate statistical analyses performed?

4. Are sensitivity analyses used?

Are the results important?

1. Was the effect strong?

a. Was the odds ratio (OR) large?

b. Were the results reported in a clinically meaningful manner, such as the absolute difference or the number needed to treat (NNT)?

2. Are the results likely to be reproducible and generalizable?

Yes

3. Were all clinically important consequences considered?

Yes

4. Are the benefits worth the harm and costs? inclusion of this gray literature into a meta-analysis is still controversial. In 150 meta-analyses from January 1989 to February 1991, most investigators had searched for unpublished material, although only $31 \%$ included this material. ${ }^{36}$ It appears the best approach is to carry out an extensive search for unpublished data and obtain them, if possible. The subsequent analysis should then be performed with and without the unpublished data; if the conclusions change based on the inclusion or exclusion of this data, the results of either approach should be viewed cautiously.

Restricting the literature search to English language only can also introduce a "Tower of Babel" bias. ${ }^{37}$ Meta-analyses published in English language journals often restrict their search to the English language. ${ }^{29}$ Investigators working in a nonEnglish-speaking country might be more likely to report positive findings in an international, English language journal and negative findings in their lo- 


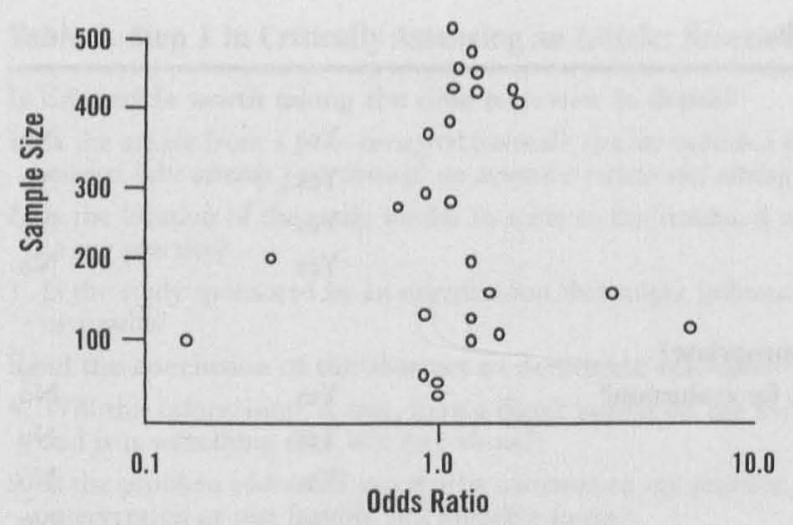

Figure 2. Funnel plot of the odds ratio versus sample size for studies included in the meta-analysis.

cal, non-English language journal. To reduce this bias, the best meta-analyses will not place a limit on the type of language searched. ${ }^{38}$

Funnel plots, which are simple scatter plots of the odds ratios of the trials against their sample size, are useful to detect publication bias in a metaanalysis. Typically, results from small studies will scatter widely at the bottom of the graph; this spread will narrow as precision increases among larger studies. ${ }^{29,39}$ In the absence of bias, the plot should resemble a symmetrical inverted funnel, as shown in Figure 2.

In the article you are assessing, you find the authors focused on RCTs, comparing different durations of antibiotic treatment in children with a diagnosis of acute otitis media. They used four different databases (MEDLINE, EMBASE, Current Contents, and Science Citation Index) with no language restrictions, and reviewed the reference lists of relevant articles to find further trials. Key words that were used are listed. The authors do not specifically mention looking at the gray literature. Although not mentioned in the Methods section, you do find later in the article that the authors address the possibility of publication bias in their search by doing a funnel plot. You are assured that the author's performed a thorough literature search and proceed with your assessment.

2. Was the method for selecting articles clear, systematic, and appropriate? Once a comprehensive literature search has been done, the investigators should have a systematic way to evaluate which of the articles should be included in the analysis. Eligibility criteria should be clearly documented in the protocol. The population should be well defined and the intervention and all outcomes clearly de- scribed. When the eligibility criteria for a metaanalysis are strict, the final number of studies included in the analysis are much fewer in comparison with the number of studies found in the literature search. ${ }^{18}$ To improve the selection process, at least two independent selectors should be used, and an evaluation should be performed on how closely they agreed on which articles should be selected and which ones should be discarded. A log should be maintained of the excluded articles to allow the reader to assess the quality of the screening tool.

In the Methods section, you read that the investigators clearly described the eligibility criteria (age 4 weeks to 18 years, clinical diagnosis of acute otitis media not on antibiotics, randomized to less than or greater than 7 days of treatment, and an assessment of clinical resolution). Using these strict criteria for selection, 7 investigators independently evaluated those articles found in the literature search. They then applied a statistical test (kappa statistic) to document this close agreement. You answer yes to this question.

3. Was the quality of the primary studies evaluated? The criteria used to reject articles as flawed must be explicit and independent of the results of those trials. The investigators should have a list of criteria, including generic (common to all research studies) and particular (specific to the area in question) aspects of quality, used to judge each trial. All the articles should have the same standard applied. Ideally, investigators should consider including only controlled trials with proper randomization of patients in a double-blinded manner who are analyzed on an intention-to-treat basis using objective measures of outcomes. ${ }^{14}$

Assessing the quality of a study can be subjective unless strict quality criteria are used, such as those found in the Jadad scale. ${ }^{40}$ Also, to decrease the possibility of bias, there should be at least two independent evaluators, and interrater agreement should be assessed. To obtain more consistent quality scores of the studies, the investigators should evaluate the articles blindly (ie, remove such information as authors, location of study, and names of the journals). ${ }^{41}$

In the article you are critiquing, the investigators used an accepted quality score (the Jadad scale) and applied this standard to all articles. At least two independent investigators assessed the quality of the articles, and there was a high degree of inter- 


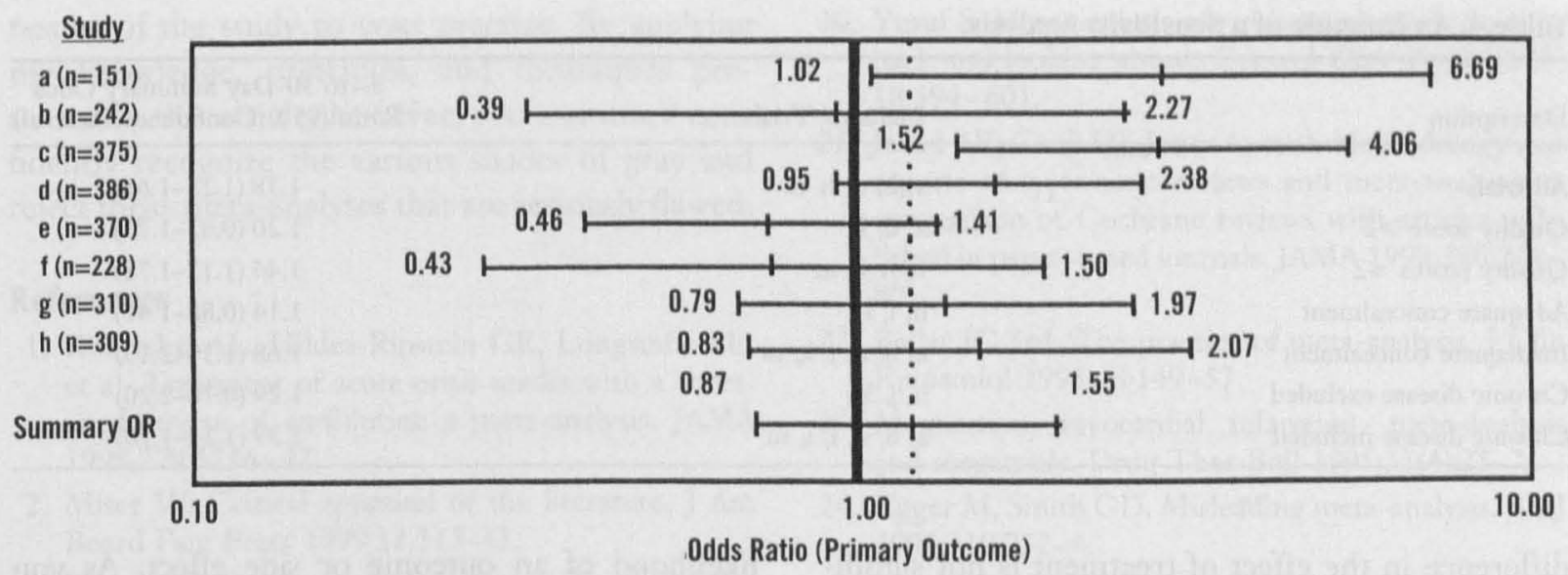

Figure 3. Graphic display of treatment outcomes (odds ratio) and $95 \%$ confidence intervals. An odds ratio greater than 1 favors treatment.

rater agreement. The majority of trials were blindly evaluated. Of the 41 articles originally selected, 12 were excluded. Reasons for their exclusion are outlined. Also of interest, you note that the overall quality score for the RCTs for treating acute otitis media is 2.7 out of 5 possible points, which suggests that quality studies on such a common condition are lacking. You answer yes to this question.

4. Were results from the studies combined appropriately? Once the articles are selected and their quality assessed, the next step in a meta-analysis involves combining the data from these studies. The studies should be similar enough in terms of design, population, and outcomes to permit the data to be combined. If the end-points studied are binary (eg, relapse versus no relapse, survival versus death, etc), odds ratios are often calculated. The odds ratio, an estimate of relative risk, has mathematical properties that allow one to combine the data easily and to test for the overall effect of significance. ${ }^{14}$

It would be inappropriate to combine the results if the studies differed greatly. A test for heterogeneity (dissimilarity) across the studies allows one to examine statistically the degree of similarity in the outcomes of the studies. Averaging the odds ratios from all the studies would give misleading results, as smaller studies are more subject to chance. To acknowledge the variability of the results between studies, and to give larger trials more influence in the overall result, meta-analyses use one of two different techniques to test for heterogeneity. The fixed-effects model regards the variability between studies to be due to random variation. Thus, if all the studies were large enough, they would yield the same results. In contrast, the random-effects model considers each study to be from a different population, the rate varies from study to study, and their differences are due to experimental error and to differences in the populations. ${ }^{16}$

You see in Table 1 of the article you are assessing that the investigators grouped the studies by the pharmacokinetic behavior of the antibiotic. They performed a test for heterogeneity (random effects) and found that the studies were similar, and the results could be combined. You answer yes to this question and proceed with your assessment.

5. Was a statistical combination (meta-analysis) done properly? The next step is the actual statistical combination (meta-analysis) of the data. The investigators should address the possibility that chance differences did not occur (a test for homogeneity). Homogeneity means that the results of each individual trial are mathematically compatible with the results of any of the others. ${ }^{11}$

Homogeneity can be quickly assessed by reviewing a graphic display of the numerical data with their odds ratios and $95 \%$ confidence intervals (Figure 3). The horizontal line for each trial shows the odds of successful treatment. A vertical line in the middle of each of these lines represents the point estimate of the difference between the groups. The width of each line represents the $95 \%$ confidence interval of this point estimate (the true answer will fall within the boundaries $95 \%$ of the time). A bold vertical line (odds ratio of 1.0) is known as the line of no effect. When the confidence interval of the result (the horizontal line) crosses the line of no effect (vertical line), then the 
Table 4. An Example of a Sensitivity Analysis.

\begin{tabular}{llc}
\hline Description & Included Trials & $\begin{array}{c}\text { 8- to 30-Day Summary Odds } \\
\text { Ratio (95\% Confidence Interval) }\end{array}$ \\
\hline All trials & $\mathrm{a}, \mathrm{b}, \mathrm{d}, \mathrm{h}-\mathrm{m}$ & $1.38(1.15-1.66)$ \\
Quality score $>2$ & $\mathrm{a}, \mathrm{d}, \mathrm{l}$ & $1.20(0.83-1.75)$ \\
Quality scores $\leq 2$ & $\mathrm{~b}, \mathrm{~h}-\mathrm{k}, \mathrm{m}$ & $1.44(1.17-1.78)$ \\
Adequate concealment & $\mathrm{h}, \mathrm{i}, \mathrm{l}$ & $1.14(0.88-1.47)$ \\
Inadequate concealment & $\mathrm{a}, \mathrm{b}, \mathrm{d}, \mathrm{j}, \mathrm{k}, \mathrm{m}$ & $1.68(1.39-2.19)$ \\
Chronic disease excluded & $\mathrm{h}, \mathrm{j}, \mathrm{k}$ & $1.29(0.76-2.20)$ \\
Chronic disease included & $\mathrm{a}, \mathrm{b}, \mathrm{d}, \mathrm{i}, \mathrm{l}, \mathrm{m}$ & $1.39(1.15-1.70)$ \\
\hline
\end{tabular}

difference in the effect of treatment is not significant at $P>.05$. In Figure 3, the confidence intervals of all but two of the trials $(a, c)$ cross this line, indicating that the treatment effects were not significant. The summary odds ratio, using either the fixed-effects or random-effects model, is also found in Figure 3. The vertical dashed line crosses the horizontal lines of all individual studies except one (c), which indicates a fairly homogeneous set of studies.

In addition to a test for homogeneity, the investigators should also perform a sensitivity analysis (Table 4). Depending on the test chosen, the same set of data may be combined to give different conclusions. If one finds that fiddling with the data in various ways makes little or no difference to the review's overall results, one can assume that the review's conclusions are accurate. In the sensitivity analysis used as an example in Table 4, the data were analyzed based on quality score, adequacy of treatment allocation, and presence or absence of chronic disease.

In Figure 1 of the article you are reviewing, you find a test for homogeneity. The investigators also used a statistical test (Peto fixed-effects model) that allowed them to calculate the summary odds ratio. They also performed a sensitivity analysis by excluding trials of lower methodological quality, trials involving patients with recurrent or chronic otitis media, and trials comparing different antibiotics; none made a difference in the overall results. Assured that the investigators appropriately performed the meta-analysis, you answer yes.

6. Are the results important? This final question forces you to consider the cost benefit and potential harm of the therapy. The investigators should show a strong effect manifested by a large ( 2.0 or greater) odds ratio. They should also address the number needed to treat, which takes into consideration the likelihood of an outcome or side effect. As you review this question, you would also like to see that all clinically important consequences are considered and that the benefits are worth the harm and costs.

In the meta-analysis you are evaluating, the odds ratio was not large, which means that there was no statistical difference in treating a child with a shorter or longer course of antibiotics. You see that you would have to treat 44 children with a 10 -day course to prevent one treatment failure at 30 days using a shorter course of antibiotics.

\section{Conclusion of Case}

After a thorough assessment of this article, you conclude it is well designed with valid results. You now feel confident that a shorter course of antibiotics for children with uncomplicated acute otitis media is just as beneficial as a longer course. As you institute the short-course antibiotic treatment as a standard within your practice, however, you also make a commitment to monitor its benefits and risks to your patients and to scan the literature for future articles that might offer additional information about length of therapy for acute otitis media. Consistency of the results in your practice, as well as across multiple published studies, is one characteristic of the scientific process that leads to acceptance and implementation.

\section{A Final Word}

With some practice and the use of Table 3, one can quickly (within a few minutes) perform a critical assessment of a meta-analysis. While performing this appraisal, it is important to keep in mind that few meta-analyses will be perfect. Only you can answer for yourself the exact shade of gray that you are willing to accept when deciding to apply the 
results of the study to your practice. By applying the knowledge, principles, and techniques presented in this article, however, you can more confidently recognize the various shades of gray and reject those meta-analyses that are seriously flawed.

\section{References}

1. Kozyrskyj AL, Hildes-Ripstein GE, Longstaffe SE, et al. Treatment of acute otitis media with a shortened course of antibiotics: a meta-analysis. JAMA 1998;279:1736-42.

2. Miser W. Critical appraisal of the literature. J Am Board Fam Pract 1999;12:315-33.

3. Pearson K. Report on certain enteric fever inoculation statistics. Br Med J 1904;3:1243-6.

4. Glass GV. Primary, secondary and meta-analysis of research. Educational Researcher 1976;5(10):3-8.

5. Chalmers TC, Matta RJ, Smith H Jr, Kunzler AM. Evidence favoring the use of anticoagulants in the hospital phase of acute myocardial infarction. N Engl J Med 1977;297:1091-6.

6. Egger M, Smith GD. Meta-analysis. Potentials and promise. BMJ 1997;315:1371-4.

7. Oxman $A D$, Guyatt $G H$. Guidelines for reading literature reviews. CMAJ 1988;138:697-703.

8. Shaughnessy AF, Slawson DC. Getting the most from review articles: a guide for readers and writers. Am Fam Physician 1997;55:2155-60.

9: Goodman SN. Have you ever meta-analysis you didn't like? Ann Intern Med 1991;114:244-6.

10. Sacks HS, Berrier J, Reitman D, Ancona-Berk VA, Chalmers TC. Meta-analyses of randomized clinical trials. N Engl J Med 1987;316:450-5.

11. Greenhalgh T. Papers that summarise other papers (systematic reviews and meta-analyses). BMJ 1997; 315:672-5.

12. Thompson SG, Pocock SJ. Can meta-analyses be trusted? Lancet 1991;338:1127-30.

13. Peipert JF, Bracken MB. Systematic reviews of medical evidence: the use of meta-analysis in obstetrics and gynecology. Obstet Gynecol 1997;89:628-33.

14. Egger M, Smith GD, Phillips AN. Meta-analysis: principles and procedures. BMJ 1997;315:1533-7.

15. Kassirer JP. Clinical trials and meta-analysis. What do they do for us? N Engl J Med 1992;327:273-4.

16. Sacks HS, Reitman D, Pagano D, Kupelnick B. Meta-analysis: an update. Mt Sinai J Med 1996;63: 216-24.

17. Egger M, Schneider M, Davey-Smith G. Spurious precision? Meta-analysis of observational studies. 1998;316:140-4.

18. Petitti DB. Meta-analysis and endocrinology. Endocrinol Metab Clin North Am 1997;26:31-44.

19. Moher D, Dulberg CS, Wells GA. Statistical power, sample size, and their reporting in randomized controlled trials. JAMA 1994;272:122-4.
20. Yusuf S. Meta-analysis of randomized trials: looking back and looking ahead. Control Clin Trials 1997; 18:594-601.

21. Jadad AR, Cook DJ, Jones A, et al. Methodology and reports of systematic reviews and meta-analyses: a comparison of Cochrane reviews with articles published in paper-based journals. JAMA 1998;280:27880.

22. Bailar JC 3rd. The practice of meta-analysis. J Clin Epidemiol 1995;48:149-57.

23. Magnesium, myocardial infarction, meta-analysis and megatrials. Drug Ther Bull 1995;33(4):25-7.

24. Egger M, Smith GD. Misleading meta-analysis. BMJ 1995;310:752-4.

25. Greenhalgh T. The MEDLINE database. BMJ 1997;315:180-3.

26. Dickersin K, Scherer R, Lefebvre C. Identifying relevant studies for systematic reviews. BMJ 1994; 309:1286-91.

27. Fox RN, Ventura MR. Efficiency of automated literature search mechanisms. Nurs Res 1984; 33:174-7.

28. Hersh WR, Hickam DH. How well do physicians use electronic information retrieval systems? A framework for investigation and systematic review. JAMA 1998;280:1347-52.

29. Egger $M$, Smith GD. Bias in location and selection of studies. BMJ 1998;316:61-6.

30. Chalmers TC, Frank CS, Reitman D. Minimizing the three stages of publication bias. JAMA 1990;263: 1392-5.

31. Rennie D, Flanagin A. Publication bias. The triumph of hope over experience. JAMA 1992;267: 411-2.

32. Scherer RW, Dickersin K, Langenberg P. Full publication of results initially presented in abstracts. A meta-analysis. JAMA 1994;272:158-62.

33. Dickersin K, Min YI, Meinert CL. Factors influencing publication of research results. Follow-up of applications submitted to two institutional review boards. JAMA 1992;267:374-8.

34. Stern JM, Simes RJ. Publication bias: evidence of delayed publication in a cohort study of clinical research projects. BMJ 1997;315:640-5.

35. Davey-Smith G, Egger M. Meta-analysis. Unresolved issues and future developments. BMJ 1998; 316:221-5.

36. Cook DJ, Guyatt GH, Ryan G, et al. Should unpublished data be included in meta-analyses? Current convictions and controversies. JAMA 1993;269: 2749-53.

37. Gregoire G, Derderian F, LeLorier J. Selecting the language of publications included in a meta-analysis: is there a Tower of Babel bias? J Clin Epidemiol 1995;48:159-63.

38. Moher D, Fortin P, Jadad AR, et al. Completeness of reporting of trials published in languages other than 
English: implications for conduct and reporting of systematic reviews. Lancet 1996;347:363-6.

39. Egger M, Davey-Smith G, Schneider M, Minder C. Bias in meta-analysis detected by a simple, graphical test. BMJ 1997;315:629-34.

40. Moher D, Jadad AR, Nichol G, Penman M, Tugwell
P, Walsh S. Assessing the quality of randomized controlled trials: an annotated bibliography of scales and checklists. Control Clin Trials 1995;16:62-73.

41. Jadad AR, Moore RA, Carroll D, et al. Assessing the quality of reports of randomized clinical trials: is blinding necessary? Control Clin Trials 1996;17:1-12. 